Article

\title{
Photo-Thermal Effects in 1D Gratings of Gold Nanoparticles
}

\author{
Giovanna Palermo ${ }^{1,2, *}$, Tiziana Ritacco ${ }^{1,2}$, Dante Maria Aceti ${ }^{1}$, Luigia Pezzi ${ }^{1}$, \\ Michele Giocondo ${ }^{2}$ and Antonio De Luca ${ }^{1,2, *}$ \\ 1 Dipartimento di Fisica, Università della Calabria, Ponte Pietro Bucci Cubo 31/C, 87036 Rende (CS), Italy; \\ tiziana.ritacco@unical.it (T.R.); dma92@hotmail.it (D.M.A.); luigia.pezzi@fis.unical.it (L.P.) \\ 2 Consiglio Nazionale delle Ricerche, Istituto di Nanotecnologia, Nanotec, SS di Cosenza, \\ Ponte Pietro Bucci Cubo 31/C, 87036 Rende (CS), Italy; michele.giocondo@cnr.it \\ * Correspondence: giovanna.palermo@fis.unical.it (G.P.); antonio.deluca@fis.unical.it (A.D.L.); \\ Tel.: +39-0984-496124 (G.P. \& A.D.L.); Fax: +39-0984-494401 (G.P. \& A.D.L.)
}

Academic Editor: Johan Wouters

Received: 14 December 2016; Accepted: 4 January 2017; Published: 6 January 2017

\begin{abstract}
This work investigates the heat delivered by a mono-layer 1D grating of gold nanoparticles (GNPs) created by photo-reduction through two-photon direct laser writing (2P-DLW) in a poly-vinyl alcohol (PVA) matrix doped with $\mathrm{HAuCl}_{4}$, under resonant laser radiation. We drop cast a film of a PVA $+\mathrm{HAuCl}_{4}$ mixture onto a glass substrate, in which we create gratings of $1 \mathrm{~mm}^{2}$ made by stripes of GNPs characterized by high polydispersivity. We demonstrate that, by controlling the pitch of the GNP stripes, we obtain different values of the photo-induced temperature variations. In the framework of thermo-plasmonics, the experimental investigation of the heat generation from a monolayer of gold nanoparticles represents a key element as a starting point to design thermo-smart platforms for sensing, solar energy harvesting and thermo-catalysis.
\end{abstract}

Keywords: thermo-plasmonics; gold nanoparticles; heat

\section{Introduction}

In recent years, the laser-nanostructure interaction has become of great interest due to the application of NPs in different fields, such as nano-optics and nano-electronics [1-4], laser nano-biomedicine [5-8] and catalysis [9,10]. This is due to the well-known property of metallic NPs called localized surface plasmon resonance (LSPR), in which the conduction band electrons oscillate coherently with the electric field of the incident light. The frequency and intensity of the LSPR absorption bands are characteristic of the material (gold, silver or platinum) and are highly sensitive to the size, distribution, and shape of the nanostructures, as well as to the environments which surround them $[11,12]$.

Optical excitation and non-radiative relaxation of the LSPR create a photo-thermal effect due to the conversion of light energy into heat. Within this LSPR-mediated heating process, the light absorbed by the nanoparticle generates a non-equilibrium electron distribution that decays via electron-electron scattering. The hot electron gas equilibrates with lattice phonons which transfer this energy into the surrounding medium, resulting in a local temperature increase [13]. The total energy transfer process is rapid $\left(10^{-10}-10^{-12} \mathrm{~s}\right)$ and efficient [14].

This effect, renamed the photo-thermal effect in plasmonic nanoparticles, has already found applications in cancer therapy [15,16], optofluidic control [17], nano-welding [18], optical data storage [19], imaging [20] and sensing [21].

Recent advances in both bottom-up and top-down fabrication techniques offer a tremendous variety of metallic NPs with numerous geometries [22] which can be immobilized on a flat substrate 
with a resolution down to a few tens of nanometers. These substrates, if appropriately designed, can be used as thermo-smart platforms, able to generate a desired value of temperature by controlling the number of GNPs photo-excited at the same time.

In the field of additive manufacturing, two-photon direct laser writing (2P-DLW) represents the most advanced optical technique for the fabrication of 2D and 3D arbitrary structures, with resolutions well below the diffraction limit [23]. Focusing a femto-second infrared laser $(\lambda=780 \mathrm{~nm}$, pulse duration of 100 femtosecond ( $\mathrm{fs}$ ) on a polymeric matrix doped with a photosensitive metal precursor, it is possible to create NPs inside the focus figure ("voxel" in a volume) through the photo-reduction of the metallic element in the precursor.

The $\mathrm{Au}^{0}$ nanoparticles were formed by the direct photo-reduction of $\mathrm{AuCl}_{4}^{-}$, according to the mechanism reported in References [24,25]. Briefly, during the irradiation process, the excited $\mathrm{Au}^{3+}$ forms the caged $\mathrm{Au}^{2+}$ complex (Equations (1) and (2)). This complex then dissociates (Equation (3)) and the unstable $\mathrm{Au}^{2+}$ quickly disproportionates to form $\mathrm{Au}^{+}$and $\mathrm{Au}^{3+}$ (Equation (4)). $\mathrm{Au}^{+}$absorbs another photon to form $\mathrm{Au}^{0}$ (Equation (5)). $\mathrm{Au}^{0}$ associates to form nuclei and the AuNPs (Equation (6)):

$$
\begin{gathered}
\left(\mathrm{HAu}^{3+} \mathrm{Cl}_{4}\right) \stackrel{h v}{\rightarrow}\left(\mathrm{HAu}^{3+} \mathrm{Cl}_{4}\right)^{*} \\
\left(\mathrm{HAu}^{3+} \mathrm{Cl}_{4}\right)^{*} \rightarrow\left(\mathrm{HAu}^{2+} \mathrm{Cl}_{3}-\mathrm{Cl}\right) \\
\left(\mathrm{HAu}^{2+} \mathrm{Cl}_{3}-\mathrm{Cl}\right) \rightarrow \mathrm{HAu}^{2+} \mathrm{Cl}_{3}-\mathrm{Cl} \\
2 \mathrm{HAu}^{2+} \mathrm{Cl}_{3} \rightarrow \mathrm{HAu}^{3+} \mathrm{Cl}_{4}+\mathrm{HAu}+\mathrm{Cl}_{2} \\
\mathrm{HAu}^{+} \mathrm{Cl}_{2} \stackrel{h v}{\rightarrow} \mathrm{HAu}^{0}+\mathrm{HCl}+\mathrm{Cl} \\
n \mathrm{Au}^{0} \stackrel{h v}{\rightarrow}\left(\mathrm{Au}^{0}\right)_{n}
\end{gathered}
$$

While the process of photo-reduction of a silver precursor (e.g., $\mathrm{AgNO}_{3}$ ) leads to the oxidation of the polymeric matrix, the photo-reduction of $\mathrm{HAuCl}_{4}$ does not chemically change the matrix. The photo-reduction of GNPs, in fact, is not promoted by the oxidation of the PVA [24].

Recent studies demonstrated the possibility to create stripes at the submicron scale, rich in gold nanoparticles, in a poly-vinyl alcohol (PVA) matrix doped with $\mathrm{HAuCl}_{4}$, by means of this 2P-DLW technique [26].

In the present study, we realized four different samples of GNP stripes spaced by $0.5,2,10$ and $100 \mu \mathrm{m}$. The thermal response of each sample, characterized by a different grating periodicity of GNPs, was monitored by means of a thermographic camera as a function of the impinging intensity of a continuous beam laser $(\lambda=532 \mathrm{~nm})$. In this way, a careful analysis of the macroscopic photo-induced heat generation of this particular 1D arrangement of GNPs was carried out.

\section{Results and Discussions}

A surface of $1 \mathrm{~mm}^{2}$ of a glass substrate was structured with a mono-dimensional grating of GNPs, direct laser-written in dried films of PVA and $\mathrm{HAuCl}_{4}$. According to the procedure described in Reference [27], the samples were developed in $\mathrm{H}_{2} \mathrm{O}$ to remove all the unexposed material, leaving only the stripes of GNPs sticking to the glass substrate.

The width of each GNP stripe, obtained by using a laser power of $25 \mathrm{~mW}$ and a scanning speed of $200 \mu \mathrm{m} / \mathrm{s}$, was of the order of $1.5 \mu \mathrm{m}$. Then we decided to design four gratings with different periods in order to control the number of GNPs that were excited at the same time by the resonant radiation; in particular, we chose a periodicity between the stripes of $0.5,2,10$ and $100 \mu \mathrm{m}$. The four samples were first analyzed by means of an optical microscope and then by an atomic force microscope (AFM).

Optical images were acquired through a $50 \times$ and $100 \times$ objective, between crossed polarizers, which allow evidencing the macroscopic response of gold nanoparticles and identifying the different 
gratings due to scattering effects (Figure 1). The sample with the period of $0.5 \mu \mathrm{m}$, in Figure 1a, appears as a dense square of GNPs, which does not show any pattern inside. On the other hand, the grating structure is evident in the samples with the other designed periods; thus, we obtained stripes rich in GNPs, spaced from $0.5 \mu \mathrm{m}$ (Figure 1b) to $100 \mu \mathrm{m}$ (Figure 1d).
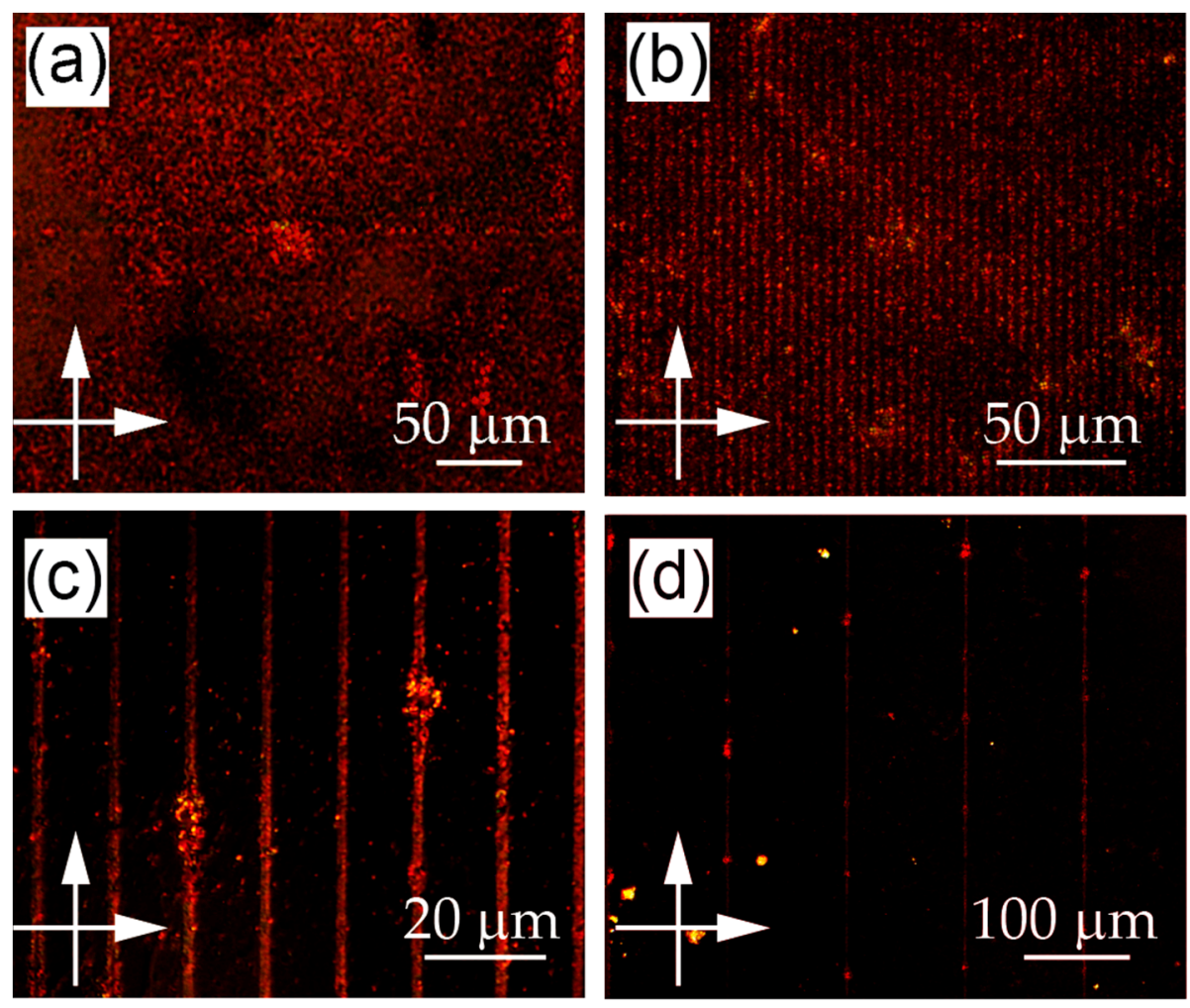

Figure 1. Microscope optical images, acquired between crossed polarizers, of the GNP gratings with period of (a) $0.5 \mu \mathrm{m}$; (b) $2 \mu \mathrm{m}$; (c) $10 \mu \mathrm{m}$; (d) $100 \mu \mathrm{m}$.

AFM images (Figure 2a) confirm the obtained arrangement of GNPs in vertical stripes, showing that the diameter of the nanoparticles, depending on the local concentration of the gold precursor and the energy dose delivered by the laser, varied over two orders of magnitude, with the largest value of the order of $100 \mathrm{~nm}$. However, for all the samples we observed an average height of approximately $40 \mathrm{~nm}$, measured by the AFM observation.

It is worth noting that the GNPs with a diameter of the order of $100 \mathrm{~nm}$ are due to a greater local concentration of gold ions; therefore, this effect was less visible in the stripes from $0.5 \mu \mathrm{m}$ to $2 \mu \mathrm{m}$ where the highest concentration of gold ions was photo-reduced in the first path of the writing beam. This led to a consequent creation of smaller GNPs, characterized by a lower polydispersivity.

In order to estimate the average size of the GNPs, a testing sample with only one stripe was prepared, obtained with the same energy dose used for the fabrications of the other samples. Scanning electron microscopy analysis was performed on this sample, characterized by a length of $1 \mathrm{~mm}$ and a width of $1.5 \mu \mathrm{m}$ (Figure 2e). By using the same write-parameter (energy dose) we are sure to obtain a stripe characterized by the same number of GNPs. By means of Image-J software [28] we counted and determined the size distribution of the GNPs in this stripe, obtaining a density value of $90 \mu^{-2}$. This value was used to calculate the density of the GNPs in each sample.

The statistical analysis confirms that all the samples exhibited an average NP diameter of approximately $40 \mathrm{~nm}$. Moreover, they presented a surface density of $1.8 \times 10^{8}, 4.5 \times 10^{7}$, $9 \times 10^{6}$ and $9 \times 10^{5} \mathrm{~mm}^{-2}$, respectively, for the samples with grating periods of $0.5,2,10$ and $100 \mu \mathrm{m}$. 

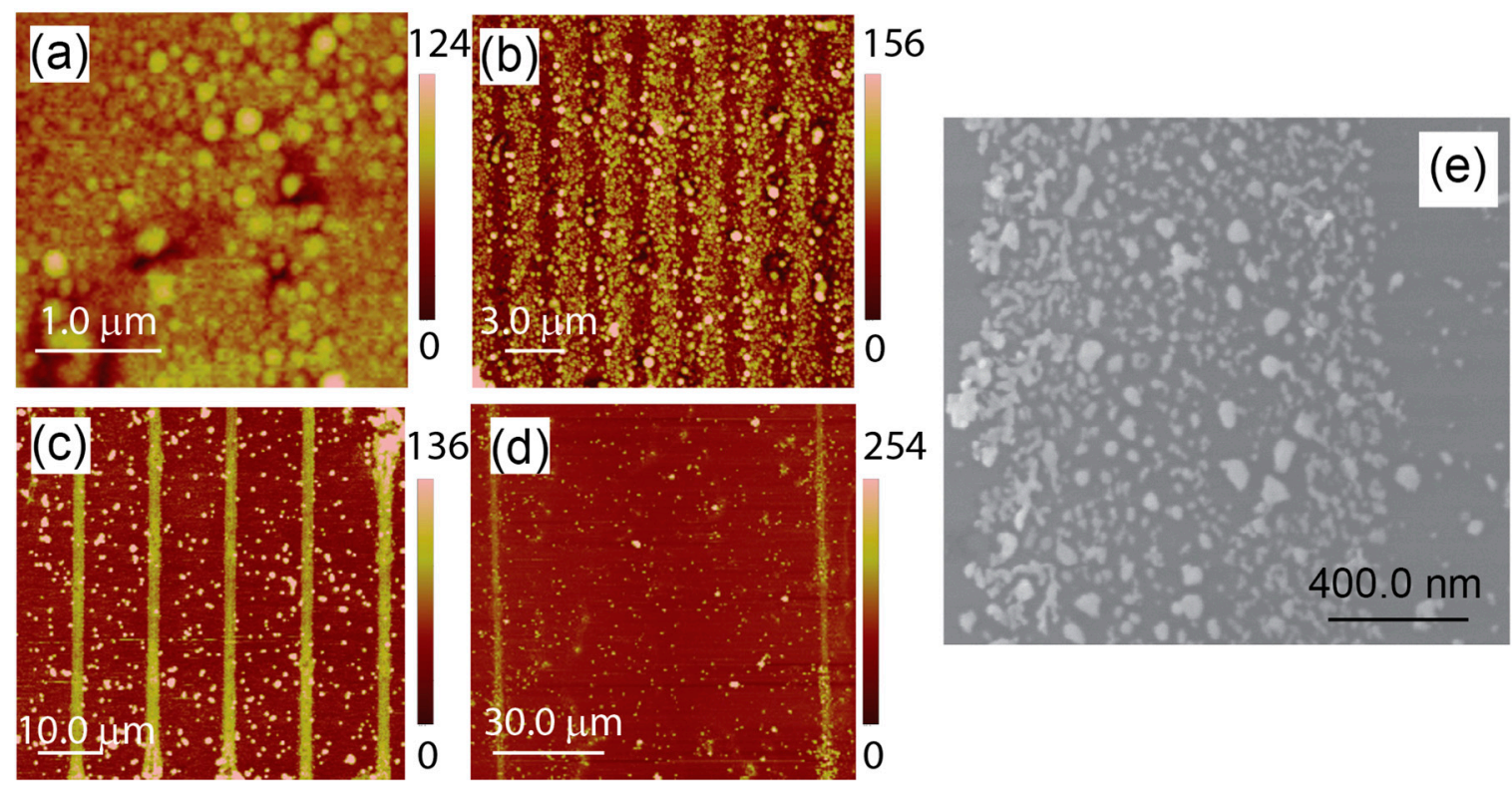

Figure 2. Atomic force microscopy images of GNPs with period of (a) $0.5 \mu \mathrm{m}$; (b) $2 \mu \mathrm{m}$; (c) $10 \mu \mathrm{m}$; (d) $100 \mu \mathrm{m}$; (e) SEM image of GNPs distributed in one stripe.

During the direct laser writing, the two-photon absorption induced a local heating of the photo-resist, which led to the thermal photo-reduction of the metallic precursor in the area surrounding the stripes and the creation of random GNPs between them. These random nanoparticles were observed in all four samples and presented a density of $0.5 \times 10^{5} \mathrm{~mm}^{-2}$, which did not affect the total density of the samples nor give a consistent macroscopic contribution.

The absorbance spectra (Figure 3a), obtained by probing the sample with unpolarized white light (wavelength in the range of 400-900 nm) at normal incidence, exhibit the typical gold LSPR absorption band centered at $560 \mathrm{~nm}$ for the GNP grating with a period of $0.5 \mu \mathrm{m}$, at $575 \mathrm{~nm}$ for the GNP grating with a period of $2 \mu \mathrm{m}$, at $587 \mathrm{~nm}$ for the GNP grating with a period of $10 \mu \mathrm{m}$, while, in the case of the grating with a period of $100 \mu \mathrm{m}$, we could not see a plasmonic band. The broadening of the LSPR peak is due to the size dispersion of GNPs, revealing that the high density of smaller nanoparticles in the samples with a $0.5 \mu \mathrm{m}$ and $2 \mu \mathrm{m}$ period led to a blue-shift in the spectrum resonance [29].

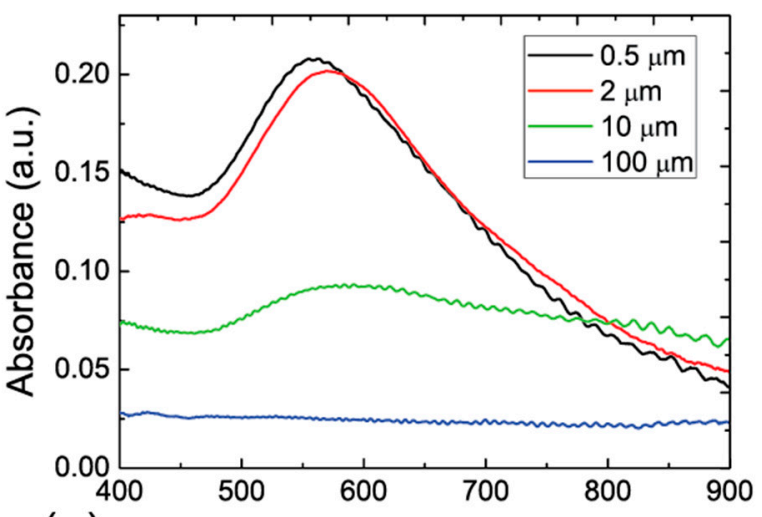

(a) Wavelength $(\mathrm{nm})$

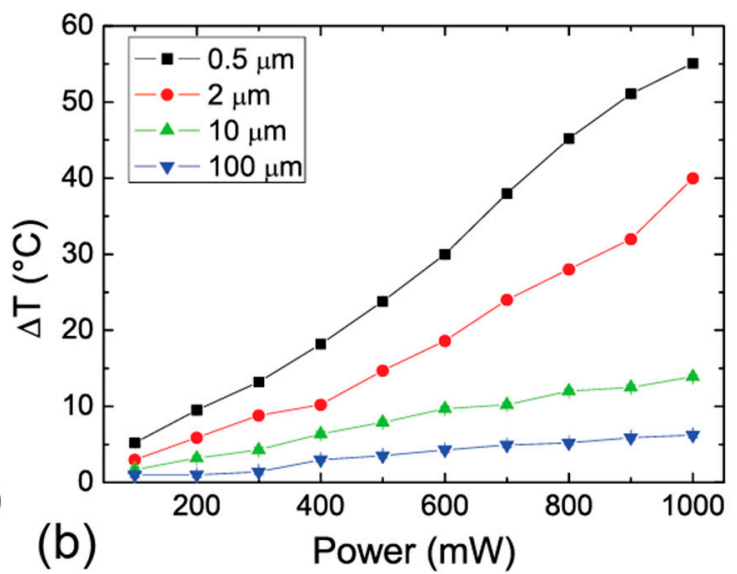

Figure 3. (a) Spectral response of the GNP substrate; (b) Temperature variations of the GNP layer for different values of the pump beam laser. 
Thermal analysis was carried out by illuminating the samples orthogonally with a continuous green laser at $\lambda=532 \mathrm{~nm}$, with a beam diameter of $(2.25 \pm 0.22) \mathrm{mm}$. Measurements were made at power intervals of $50 \mathrm{~mW}$ in the range of $50-550 \mathrm{~mW}$, corresponding to intensity variations from 1.26 to $13.84 \mathrm{~W} / \mathrm{cm}^{2}$. The photo-induced temperature variation was monitored by using a thermo-camera (E40 by FLIR) characterized by a sensitivity of $0.07^{\circ} \mathrm{C}$ and a spatial resolution of $2.72 \mathrm{mrad}$. The camera control parameters were appropriately set to consider both environmental and materials properties (room temperature, level of humidity in the room, emissivity of the material). By detecting the highest temperature value $T$, corresponding to the central pixel of each hot-spot shown in the thermographic images, it was possible to plot the characteristic temperature variation $\Delta T \equiv T-T_{0}$ as a function of the impinging power density (Figure $3 \mathrm{~b}$, here $T_{0}$ is the room temperature). A linear increase was observed in all the samples.

The measured temperature variation results were $55.1 \pm 0.3,40.0 \pm 0.3,13.9 \pm 0.3$, and $6.2 \pm 0.3^{\circ} \mathrm{C}$, respectively, for the samples with $0.5,2,10$ and $100 \mu \mathrm{m}$ grating periods.

The same values of $\Delta T$ were detected by monitoring different areas of the same sample. Morphological analysis carried out after the thermal test showed that the distribution of the NPs was not affected by the laser exposure. As we can see from experimental measurements, the temperature variations $\Delta T$ strongly depend on two parameters: (i) the GNPs' surface density and (ii) the intensity of the pump beam.

By increasing the power density of the pump beam, $T$ increased linearly until it reached saturation, while variations in the GNPs' stripes pitch resulted in a change in the P-T curve slope.

By following the fabrication protocol described in this work we can easily control the photo-induced temperature variations by acting on the number and the distance between the GNP stripes. It represents a really fast and simple way to obtain integrable, on-chip, photo-thermally active areas.

\section{Materials and Methods}

Samples preparation. The technique and materials used are described in reference [27]. Briefly, a herbium laser, with central wavelength at $780 \mathrm{~nm}$, pulse duration of $100 \mathrm{fs}$ and repetition rate of $80 \mathrm{MHz}$, is used to perform direct laser writing. The laser beam is focused on the sample through a $63 \times$ objective, with a Numerical Aperture (N.A.) equals to 1.4.The transversal position is controlled through a galvo scanner.

The sample is prepared by mixing and stirring for $24 \mathrm{~h}$ at room temperature $9.5 \mathrm{mg}$ tetrachloroauric acid tetrahydrate $\left(\mathrm{HAuCl}_{4} \cdot 3 \mathrm{H}_{2} \mathrm{O}\right)$ dissolved in $2 \mathrm{~mL}$ of $\mathrm{H}_{2} \mathrm{O}$ with a solution of $1.3 \mathrm{mg}$ PVA (Mw: $13.000-23.000 \mathrm{~g} / \mathrm{mol}$ ) in $2 \mathrm{~mL} \mathrm{H}_{2} \mathrm{O}$ (98\% hydrolyzed). The mixture, photosensitive at $400 \mathrm{~nm}$, was drop-casted on the glass substrates and left to dry for $1 \mathrm{~h}$, before the writing process.

As shown by the graphic (Figure 4a), a solution of PVA in water is not sensitive to $780 \mathrm{~nm}$ radiation. $\mathrm{HAuCl}_{4}$ can be photo-reduced if invested with UV light, but through DLW process it is possible to obtain a resolution well below the diffraction limits, due to the non-linear optical process of two photon absorption mechanism, whose cross section is proportional to the imaginary part of the susceptibility at the third order and is a process with a threshold [30]. In this way the GNPs are photo-reduced only in the focus figure of the laser beam and not in all the irradiated area (see Figure $4 b$ ).

After the 2P-DLW of the GNPs, the samples are developed in distilled water for $30 \mathrm{~min}$, the PVA, at this stage, is totally removed with all the GNPs that are not on the surface, then the samples are left to dry before any characterization.

Thermo-camera parameters. Reflected temperature was set to $T_{0}=20^{\circ} \mathrm{C}$, emissivity of glass to $\epsilon=0.83$ and the working distance between camera and object was adjusted to the allowed minimum value, i.e., less than $10 \mathrm{~cm}$. The instrument provides images of 19200 pixels $(160 \times 120 \mathrm{px})$, where each pixel represents a temperature value. 

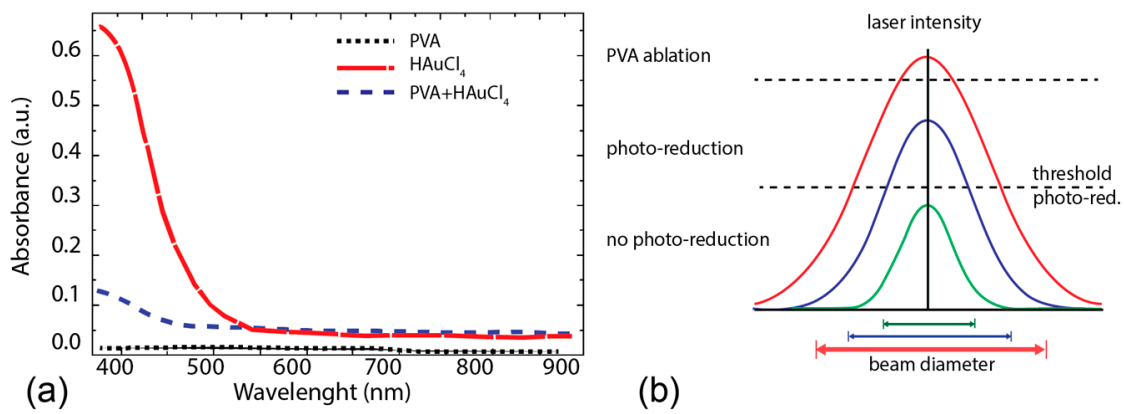

(b)

Figure 4. (a) Absorption spectra of dried films of: gold-doped PVA (blue line), $\mathrm{HAuCl}_{4}$ (red line) and PVA (black line) before the process of 2P-DLW; (b) Sketch of the phenomena involved in the volume irradiated by the laser beam.

Atomic Force Microscopy. No contact mode atomic force microscopy (AFM) (Catalyst, Bruker-Nano, Santa Barbara, CA, USA), was employed for the analysis of sample substrates by using silicon tips (radius $8 \mathrm{~nm}$ ) on a Antimony (n) doped Si lever.

Scanning Electron Microscopy. Morphological analysis was carried out by using a Quanta 400 (Hillsboro, OR, USA) by Fei Scanning Electron Microscope (SEM). The measurements were performed at 0.9 mbar and $15 \mathrm{kV}$.

Spectrometer. The spectral analysis on the samples was carried out by means of AvaSpec-ULSD2048 spectrometer by AVANTES (Apeldoorn, The Netherlands).

\section{Conclusions}

In this work a characterization of the photo-induced heat delivered by GNPs arranged in stripes onto a solid flat substrate was carried out. The samples were obtained by means of a direct laser writing technique based on a two-photon photo-reduction process, used on a PVA matrix doped with $\mathrm{HAuCl}_{4}$.

We created four 1D gratings of $1 \mathrm{~mm}^{2}$ rich in gold nanoparticles, with different periods of $0.5,2$, 10 and $100 \mu \mathrm{m}$. The morphologic characterization was carried out through AFM and SEM imaging, which allowed for measuring the density of GNPs in the four samples. Irradiating the gratings with a continuous green laser at different power values, we were able to induce a consistent temperature variation from $6.2 \pm 0.3{ }^{\circ} \mathrm{C}$ to $55.1 \pm 0.3{ }^{\circ} \mathrm{C}$.

These results demonstrated that by controlling the stripes' pitch in the obtained 1D gratings of GNPs, we could control the number of GNPs excited at the same time and the resulting macroscopic temperature variations, paving the way for a wide range of applications in many fields, such as sensing platforms, solar energy harvesting and thermo-catalysis.

Acknowledgments: The research leading to these results has received support and funding from the Italian project "Nano-Lase"-PRIN 2012, Protocol No. 2012JHFYMC. The research is supported by the CNR PON project "Material and processes BEYOND the NANO scale", Beyond Nano, cod. PONa3-00362. We acknowledge the experimental support of Giovanni Desiderio for SEM characterization.

Author Contributions: Giovanna Palermo and Tiziana Ritacco conceived and designed the experiments; Tiziana Ritacco realized the samples and performed the AFM morphological analysis; Dante Maria Aceti performed the optical morphological and spectral analysis. Giovanna Palermo and Luigia Pezzi performed the thermal test; Giovanna Palermo and Tiziana Ritacco wrote the paper. Michele Giocondo and Antonio De Luca conceived and supervised the work.

Conflicts of Interest: The authors declare no conflict of interest.

\section{References}

1. Zheludev, N. Single nanoparticle as photonic switch and optical memory element. J. Opt. A Pure Appl. Opt. 2006, 8, S1-S9. [CrossRef] 
2. Pelton, M.; Aizpurua, J.; Bryant, G. Metal-nanoparticle plasmonics. Laser Photonics Rev. 2008, 2, $136-159$. [CrossRef]

3. Sonnefraud, Y.; Leen Koh, A.; McComb, D.W.; Maier, S.A. Nanoplasmonics: Engineering and observation of localized plasmon modes. Laser Photonics Rev. 2012, 6, 277-295. [CrossRef]

4. Jin, Y.; Li, Q.; Li, G.; Chen, M.; Liu, J.; Zou, Y.; Fan, S. Enhanced optical output power of blue light-emitting diodes with quasi-aligned gold nanoparticles. Nanoscale Res. Lett. 2014, 9, 7. [CrossRef] [PubMed]

5. Halas, N.J. The photonic nanomedicine revolution: Let the human side of nanotechnology emerge. Nanomedicine 2009, 4, 369-371. [CrossRef] [PubMed]

6. Kennedy, L.C.; Bickford, L.R.; Lewinski, N.A.; Coughlin, A.J.; Hu, Y.; Day, E.S.; Drezek, R.A. A New Era for Cancer Treatment: Gold-Nanoparticle-Mediated Thermal Therapies. Small 2011, 7, 169-183. [CrossRef] [PubMed]

7. Huang, X.; Jain, P.K.; El-Sayed, I.H.; El-Sayed, M.A. Plasmonic photothermal therapy (PPTT) using gold nanoparticles. Lasers Med. Sci. 2008, 23, 217-228. [CrossRef] [PubMed]

8. Pustovalov, V.K.; Smetannikov, A.S.; Zharov, V.P. Photothermal and accompanied phenomena of selective nanophotothermolysis with gold nanoparticles and laser pulses. Laser Phys. Lett. 2008, 5, 775-792. [CrossRef]

9. Adleman, J.R.; Boyd, D.A.; Goodwin, D.G.; Psaltis, D. Heterogenous catalysis mediated by plasmon heating. Nano Lett. 2009, 9, 4417-4423. [CrossRef] [PubMed]

10. Narayanan, R.; El-Sayed, M.A. Some aspects of colloidal nanoparticle stability, catalytic activity, and recycling potential. Top. Catal. 2008, 47, 15-21. [CrossRef]

11. Goia, D.V. Preparation and formation mechanisms of uniform metallic particles in homogeneous solutions. J. Mater. Chem. 2004, 14, 451-458. [CrossRef]

12. Turkevich, J.; Stevenson, P.T.; Hillier, J. A study of the nucleation and growth processes in the synthesis of colloidal gold. Discuss. Faraday Soc. 1951, 11, 55-75. [CrossRef]

13. Palpant, B. Photothermal properties of gold nanoparticles. In Gold Nanoparticles for Physics, Biology and Chemistry, 2nd ed.; Louis, C., Pluchery, O., Eds.; Imperial College: London, UK, 2012; Volume 3, pp. 75-102.

14. Richardson, H.H.; Carlson, M.T.; Tandler, P.J.; Hernandez, P.; Govorov, A.O. Experimental and theoretical studies of light-to-heat conversion and collective heating effects in metal nanoparticle solutions. Nano Lett. 2009, 9, 1139-1146. [CrossRef] [PubMed]

15. Xiaohua, H.; El-Sayed, M.A. Plasmonic photo-thermal therapy (PPTT). Alex. J. Med. 2011, 47, 1-9.

16. Abadeer, N.S.; Murphy, C.J. Recent Progress in Cancer Thermal Therapy Using Gold Nanoparticles. J. Phys. Chem. C 2016, 120, 4691-4716. [CrossRef]

17. Liu, G.L.; Kim, J.; Lu, Y.U.; Lee, L.P. Optofluidic control using photothermal nanoparticles. Nat. Mater. 2006, 5, 27-32. [CrossRef] [PubMed]

18. Tohmyoh, H.; Imaizumi, T.; Hayashi, H.; Saka, M. Welding of Pt nanowires by Joule heating. Scr. Mater. 2007, 57, 953-956. [CrossRef]

19. Challener, W.A.; Peng, C.; Itagi, A.V.; Karns, D.; Peng, W.; Peng, Y.; Ju, G. Heat-assisted magnetic recording by a near-field transducer with efficient optical energy transfer. Nat. Photonics 2009, 3, 220-224. [CrossRef]

20. Jain, P.K.; Lee, K.S.; El-Sayed, I.H.; El-Sayed, M.A. Calculated absorption and scattering properties of gold nanoparticles of different size, shape, and composition: Applications in biological imaging and biomedicine. J. Phys. Chem. B 2006, 110, 7238-7248. [CrossRef] [PubMed]

21. Pissuwan, D.; Valenzuela, S.M.; Cortie, M.B. Therapeutic possibilities of plasmonically heated gold nanoparticles. Trends Biotechnol. 2006, 24, 62-67. [CrossRef] [PubMed]

22. Grzelczak, M.; Perez-Juste, J.; Mulvaney, P.; Liz-Marzan, L.M. Shape control in gold nanoparticle synthesis. Chem. Soc. Rev. 2008, 37, 1783-1791. [CrossRef] [PubMed]

23. Engelhardt, S. Direct Laser Writing, in Laser Technology in Biomimetics; Schmidt, V., Belegratis, M.R., Eds.; Springer: Berlin/Heidelberg, Germany, 2013; pp. 13-56.

24. Sakamoto, M.; Fujistuka, M.; Majima, T. Light as a construction tool of metal nanoparticles: Synthesis and mechanism. J. Photochem. Photobiol. C 2009, 10, 33-56. [CrossRef]

25. Pucci, A.; Bernabo, M.; Elvati, P.; Meza, L.I.; Galembeck, F.; de Paula Leite, C.A.; Ruggeri, G. Photoinduced formation of gold nanoparticles into vinyl alcohol based polymers. J. Mater. Chem. 2006, 16, 1058-1066. [CrossRef]

26. Kaneko, K.; Sun, H.B.; Duan, X.M.; Kawata, S. Two-photon photoreduction of metallic nanoparticle gratings in a polymer matrix. Appl. Phys. Lett. 2003, 83, 1426-1428. [CrossRef] 
27. Ritacco, T.; Ricciardi, L.; La Deda, M.; Giocondo, M. Controlling the optical creation of gold nanoparticles in a PVA matrix by direct laser writing. J. Eur. Opt. Soc. Rapid 2016, 11, 16008. [CrossRef]

28. Schneider, C.A.; Rasband, W.S.; Eliceiri, K.W. NIH Image to ImageJ: 25 years of image analysis. Nat. Methods 2012, 9, 671-675. [CrossRef] [PubMed]

29. Chen, H.; Kou, X.; Yang, Z.; Ni, W.; Wang, J. Shape-and size-dependent refractive index sensitivity of gold nanoparticles. Langmuir 2008, 24, 5233-5237. [CrossRef] [PubMed]

30. Passinger, S. Two-Photon Polymerization and Application to Surface Plasmon Polaritons; Cuvillier Verlag: Gottingen, Germany, 2008.

(c) 2017 by the authors; licensee MDPI, Basel, Switzerland. This article is an open access article distributed under the terms and conditions of the Creative Commons Attribution (CC-BY) license (http://creativecommons.org/licenses/by/4.0/). 